Revue

Revue de l'histoire des religions

de Ihistoire des religions

\title{
Le mythe fait-il le mystère ? Interprétations chrétiennes des mystères égyptiens ( $\mathrm{II}^{\mathrm{e}}-\mathrm{IV}^{\mathrm{e}}$ siècles)
}

Does myth make mystery? Christian interpretations of Egyptian mysteries

(Second-fourth centuries AD)

Francesco Massa

\section{(2) OpenEdition}

Édition électronique

URL : http://journals.openedition.org/rhr/9354

DOI : 10.4000/rhr.9354

ISSN : 2105-2573

Éditeur

Armand Colin

\section{Édition imprimée}

Date de publication : 1 décembre 2018

Pagination : 701-722

ISBN : 978-2-200-93200-8

ISSN : 0035-1423

Référence électronique

Francesco Massa, «Le mythe fait-il le mystère ? Interprétations chrétiennes des mystères égyptiens ("I- ${ }^{\mathrm{IV}} \mathrm{e}$ siècles) », Revue de l'histoire des religions [En ligne], 4 | 2018, mis en ligne le 01 décembre 2020, consulté le 13 janvier 2021. URL : http://journals.openedition.org/rhr/9354 ; DOI : https://doi.org/ 10.4000/rhr.9354 


\section{Le mythe fait-il le mystère ? Interprétations chrétiennes des mystères égyptiens $\left(\mathrm{II}^{\mathrm{e}}-\mathrm{IV}^{\mathrm{e}} \text { siècles }\right)^{1}$}

L'article examine les textes des apologètes chrétiens sur ce que l'on appelle les "mystères égyptiens » à l'époque impériale. Les sources sur ce sujet étant limitées, les témoignages de Minucius Félix, Lactance et Firmicus Maternus constituent des documents précieux pour étudier les mystères d'Isis et d'Osiris. D'après ces auteurs, ces mystères ne seraient qu'une illustration rituelle des récits mythiques relatifs à la quête d'Isis. L'idée d'une imitation du mythe par les célébrations mystériques est absente de la plupart des sources païennes sur les mystères égyptiens. Il semble que les auteurs chrétiens se servent en fait du modèle éleusinien, et de son mythe étiologique, qu'ils appliquent aux mystères égyptiens, faisant ainsi de ces "mystères » une catégorie cohérente.

Does myth make mystery?

Christian interpretations of Egyptian mysteries (Second-fourth centuries AD)

The article examines the evidence provided by Christian apologists on what is referred to as "Egyptian mysteries" in the Imperial period. Our sources on this topic are limited, and the evidence provided by Minucius Felix, Lactantius, and Firmicus Maternus constitute precious documents for the study of the mysteries of Isis and Osiris. According to these authors, Egyptian mysteries are merely a ritual illustration of the mythical narratives on the quest of Isis. The notion that mystery rituals imitate myth is however absent from most ancient pagan sources addressing Egyptian mysteries. It seems that Christian authors in fact used the Eleusinian model, and its etiological myth, to describe Egyptian mysteries, giving to the latter category a wholly homogenous character.

1. L'article s'inscrit dans un projet de recherche triennal (2015-2018), financé par le Fonds National Suisse de la recherche scientifique: "Des mystères grecs et romains aux mystères chrétiens ? Un nouveau regard sur les contacts religieux du monde impérial romain?» (www.unige.ch/lettres/antic/unites/hr/projet-fnsambizione/). Ce travail n'aurait pas pu voir le jour sans les réflexions menées dans le groupe de recherche "Les cultes à mystères (mystèria, teletai, orgia, etc.) et leurs acteurs spécialisés » que Nicole Belayche et moi-même pilotons au sein de l'UMR 8210 AnHiMA (www.anhima.fr/spip.php?article1164). Je tiens à remercier vivement Laurent Bricault et Youri Volokhine pour leurs relectures et suggestions, qui m'ont permis d'améliorer mon texte. 
Quel rôle jouait le "mythe » dans les cultes que l'on dit «à mystères »? Les récits mythiques sur les dieux influençaientils et façonnaient-ils la célébration des pratiques mystériques ? La question est complexe et soulève de nombreux problèmes d'interprétation, à partir de la construction des catégories modernes du «mythe» et du «rite», sans doute les notions les plus débattues dans le domaine de l'histoire des religions ${ }^{2}$. Dans les pages qui suivent, on se concentrera sur un aspect spécifique de cette problématique, à savoir le rôle des récits mythiques relatifs à Isis et Osiris dans les «mystères égyptiens ». Pour ce faire, j'analyserai quelques textes chrétiens des $\mathrm{II}^{\mathrm{e}}-\mathrm{IV}^{\mathrm{e}}$ siècles abordant les mystères égyptiens, afin de comprendre dans quelle mesure l'interprétation chrétienne des récits mythiques a influencé la perception de ces cérémonies ${ }^{3}$.

\section{LES « MYSTÈRES ÉGYPTIENS » : UNE CATÉGORIE CHRÉTIENNE?}

Les «mystères égyptiens» sont au centre de l'attention de la critique depuis longtemps, non seulement en tant que phénomène rituel antique, mais aussi pour leur réception dans le monde moderne ${ }^{4}$. Jan Assmann, par exemple, a très bien reconstruit la

2. Je ne rentrerai pas ici dans la querelle du rapport entre les deux notions modernes de "mythe » et "rite », plusieurs études ayant déjà apporté des éclaircissements importants à ce sujet: Claude Calame, "Mythe" et "rite" en Grèce : des catégories indigènes ? », Kernos, t. 4, 1991, p. 179-204 ; Jan N. Bremmer, "Myth and Ritual in Ancient Greece: Observations on a Difficult Relationship », Griechische Mythologie und frühes Christentum, éd. Raban von Haehling, Darmstadt, Wissenschaftliche Buchgesellschaft, 2005, p. 21-43 et Charles Delattre, «Construire le mythe : une perspective pragmatique »,Mythe et fiction, éd. Danièle Auger et Charles Delattre, Nanterre, Presses universitaires de Paris Ouest, 2010 (http://books.openedition.org/pupo/1802).

3. Sur le problème de la définition des mystères dans l'antiquité, voir Nicole Belayche, Francesco Massa, «Les mystères. Quelques balises introductives: lexique et historiographie », Mètis. Anthropologie des mondes grecs anciens, NS, t. 14,2016 , p. 7-19.

4. À ce propos, je renvoie aux colloques internationaux sur les études isiaques, organisés par Laurent Bricault, dont les actes sont publiés dans la collection «Religions in the Graeco-Roman World », chez Brill, et à Valentino Gasparini, 
naissance de l'idée de «mystères égyptiens » au XVIII siècle, notamment dans le contexte de la Franc-Maçonnerie ${ }^{5}$. Deux problèmes majeurs interviennent lorsqu'on se penche sur ce phénomène dans l'Antiquité. Le problème principal réside dans le corpus documentaire dont nous disposons, qui demeure assez limité. Comme l'a souligné Françoise Dunand, «la plupart des auteurs se contentent de reprendre le texte d'Apulée, dont les informations volontairement brèves et ambiguës, concernant l'initiation proprement dite, peuvent donner matière à des interprétations diverses $»^{6}$. En effet le dernier livre des Métamorphoses d'Apulée est utilisé souvent par les Modernes comme une sorte de traité sur les mystères égyptiens, bien qu'il soit important de ne pas oublier qu'il s'agit d'une œuvre littéraire et, plus particulièrement, d'un roman qui répond à des codes rhétoriques spécifiques, qui concerne des cultes qui ne conservent plus d'égyptien que des traces ${ }^{7}$. D'autres auteurs non-chrétiens utilisent la terminologie des mystères pour parler des cultes isiaques à l'époque impériale sans toutefois rentrer dans les détails de ces pratiques rituelles ${ }^{8}$.

Richard Veymiers éd., Individuals and Materials in the Graeco-Roman Cults of Isis. Agents, Images, and Practices, Proceedings of the VIth International Conference of Isis Studies, Leyde-Boston, Brill («Religions in the Graeco-Roman World », t. 187), 2018.

5. Voir par exemple Jan Assmann, L'Égypte ancienne: entre mémoire et science, Paris, Hazan, 2009, p. 83-143 («Les mystères égyptiens ») et id., Religio duplex. Comment les Lumières ont réinventé la religion des Égyptiens, Paris, Aubier, 2013 (édition originale : Berlin, 2010). Cf. aussi Christiane Zivie-Coche, Françoise Dunand éd., Die Religionen des alten Ägypten, Stuttgart, Kolhammen, 2013, en particulier p. 658-667, et Corinne Bonnet, Laurent Bricault éd., Les mille et une vies d'Isis. La réception des divinités du cercle isiaque de l'Antiquité à nos jours, Toulouse, à paraître.

6. Françoise Dunand, «Culte d'Isis ou religion isiaque?», Isis on the Nile. Egyptian Gods in Hellenistic and Roman Egypt, Proceedings of the IV ${ }^{\text {th }}$ International Conference of Isis Studies (Liège, November 27-29, 2008), éd. Laurent Bricault et Miguel John Versluys, Leyde-Boston, Brill (« Religions in the Graeco-Roman World », t. 171), 2010, p. 50-51, ici n. 67.

7. Sur le modèle apuléen, voir Richard Veymiers, «Les mystères isiaques et leurs expressions figurées. Des exégèses modernes aux allusions antiques », Figuring Mysteries in Graeco-Roman Antiquity, éd. Nicole Belayche et Francesco Massa, Leyde-Boston, Brill, à paraître. Sur les problèmes posés par le dernier livre des Métamorphoses d'Apulée, voir Wytse Hette Keulen et Ulrike EgelhaafGaiser éd., Aspects of Apuleius' Golden Ass, vol. III, The Isis Book. A Collection of Original Papers, Leyde-Boston, Brill, 2012.

8. Pour l'époque impériale, voir Plutarque, Isis et Osiris XXV, 360-361; Lucien, L'assemblée des dieux XI et Artémidore, La clé des songes II, 39. 
Le second problème concerne l'appartenance de ces «mystères égyptiens » à la catégorie plus large des «mystères » antiques ou, plus exactement, des «cultes à mystères » grecs et romains. On peut citer encore, à ce propos, un autre passage de Françoise Dunand, tiré d'un article plus ancien, qui proposait une réflexion générale et une synthèse sur les mystères égyptiens aux époques hellénistique et romaine : "tout se passe comme s'il existait une sorte de "bien commun" à tous les mystères gréco-orientaux, qui s'empruntent mutuellement rites et formules $»^{9}$.

À partir du $\mathrm{II}^{\mathrm{e}}$ siècle de notre ère, on remarque la mise en place d'un modèle mystérique. Ce modèle est souvent fondé sur l'exemple d'Éleusis qui devient le paradigme et le point de repère pour la représentation littéraire des autres cultes à mystères, dont les mystères égyptiens ${ }^{10}$. L'hypothèse que $\mathrm{j}$ 'ai avancée dans un travail précédent est que nous devons l'idée de ce «bien commun» notamment aux auteurs chrétiens qui réfléchissaient à cette époque sur la nature des religions traditionnelles de l'Empire ${ }^{11}$. Cela ne signifie pas que des auteurs païens n'aient jamais réfléchi au sens et à la valeur des mystères, et Plutarque - qui revient à maintes reprises sur ces pratiques rituelles - ou encore Strabon le montrent bien $^{12}$. Néanmoins, une analyse attentive des sources montre qu'une

9. Françoise Dunand, «Les mystères égyptiens aux époques hellénistique et romaine », Mystères et syncrétismes, éd. Françoise Dunand, Marc Philonenko et André Benoît, Strasbourg, Paul Geuthner («Études d'histoire des religions », t. 2), 1975, p. 11-62, ici p. 23.

10. Un exemple qui montre bien l'importance du modèle des mystères d'Éleusis dans la construction de nouvelles pratiques rituelles est celui du culte dédié au serpent Glycon, fondé par Alexandre d'Abonouteichos, du moins dans la représentation qu'en donne Lucien dans son Alexandre ou le faux prophète, 38. Sur la diffusion du modèle éleusinien à l'époque impériale voir Francesco Massa, «Éleusis-Rome aller/retour. Mobilités religieuses autour des mystères éleusiniens à l'époque impériale ", Migrations et mobilité religieuse. Espaces, contacts, dynamiques et interférences, éd. Bassir Amiri, Besançon, Presses Universitaires de Franche-Comté, à paraître.

11. Voir Francesco Massa, «La notion de "mystères" au II siècle de notre ère : regards païens et Christian turn », Mètis. Anthropologie des mondes grecs anciens, NS, t. 14, 2016, p. 109-132.

12. Sur Plutarque, voir notamment Peter Van Nuffelen, «Words of truth. Mystical silence as a philosophical and rhetorical tool in Plutarch », Hermatena, t. 182, 2007, p. 9-39; sur Strabon, Nicole Belayche, «Strabon historien des religions comparatiste dans sa digression sur les Courètes », Revue de l'histoire des religions, t. 234, fasc. 4, 2017, p. 613-633 et Mélanie Lozat, Une lecture de Strabon dans la perspective de l'histoire des religions, thèse soutenue à l'Université de Genève, le 27 septembre 2018, p. 239-266. 
généralisation de la catégorie de «mystères » (j'entends par là une catégorie appliquée sans distinction à Éleusis, Samothrace, Dionysos, Orphée, Isis, Cybèle, Mithra, etc.) n'est attestée qu'à partir des textes littéraires chrétiens. Dès le $\mathrm{II}^{\mathrm{e}}$ siècle, dans leurs traités polémiques et apologétiques, les auteurs chrétiens anciens n'ont cessé de souligner l'immoralité des pratiques liées aux divers cultes à mystères de l'Antiquité ${ }^{13}$; ce faisant, ils ont fait de la catégorie des «mystères » une composante fondamentale du vocabulaire religieux de l'époque. Au demeurant, il me semble que la catégorie moderne et a priori descriptive de "mystères » dépend encore largement du travail de ces apologètes chrétiens et de la définition qu'ils ont donnée de cette notion dans leurs traités polémiques.

En ce qui concerne les mystères égyptiens, il faut noter que, du moins depuis Hérodote, les Grecs ont interprété certains rituels

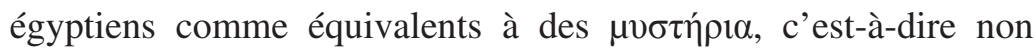
pas comme des «mystères" en général mais comme les rites éleusiniens qui, à l'époque classique, sont quasiment les seules pratiques appelées $\mu \nu \sigma \tau \eta \dot{\rho} \rho \alpha^{14}$. Lorsqu'Hérodote ou d'autres auteurs grecs de cette époque évoquent les «mystères égyptiens », ils ne font pas référence à une catégorie religieuse générale ou abstraite des «mystères », comme le feront à l'époque romaine les auteurs chrétiens. Par ailleurs, les mystères dont parle Hérodote n'ont guère en commun avec ceux d'Isis et/ou d'Osiris dont parle Apulée.

13. L'expression «cultes à mystères» s'est imposée dans la littérature scientifique notamment dans le but de s'opposer au paradigme des « religions à mystères » : cf. notamment Walter Burkert, Les cultes à mystères dans l'Antiquité, Paris, Les Belles Lettres, 1992 (édition originale Cambridge (Mass.)-Londres, 1987), p. 14-15. Pour quelques réflexions sur le débat historiographique voir Nicole Belayche, Francesco Massa, «Les mystères. Quelques balises introductives: lexique et historiographie », Mètis. Anthropologie des mondes grecs anciens, NS, t. 14,2016, p. $7-19$.

14. Voir notamment Hérodote, II, 171, 1-2. Sur les mystèria d'Osiris d'après Hérodote, voir Laurent Coulon, « Osiris chez Hérodote? », Hérodote et l'Égypte. Regards croisés sur le Livre II de l'Enquête d'Hérodote, éd. Laurent Coulon, Pascale Giovannelli-Jouanna et Flore Kimmel Clauzet, Lyon, Maison de l'Orient et de la Méditerranée, 2013, p. 167-190. 


\section{LES RÉCITS MYTHIQUES DANS LES MYSTÈRES ÉGYPTIENS}

Dans les pages qui suivent j'analyserai l'usage chrétien des mythes dans la construction de la catégorie des «mystères égyptiens » à l'époque impériale et l'idée que les rites mystériques imitent les mythes. Cette conception d'une imitation du mythe par les rites des mystères est absente des sources non-chrétiennes. Il suffit de penser au livre XI des Métamorphoses d'Apulée : à aucun moment l'initiation de Lucius n'est liée ou mise en relation avec les récits mythiques sur Isis et Osiris. En revanche, la plupart des auteurs chrétiens qui parlent des «mystères égyptiens » tissent un lien entre les pratiques rituelles et les récits du deuil et de la quête d'Isis. Mon hypothèse est qu'il s'agit d'une spécificité des interprétations chrétiennes, qui a sans doute influencé notre manière de penser la liturgie des «mystères égyptiens ».

Le seul auteur non chrétien qui laisserait entendre un rôle des mythes fondateurs dans les pratiques rituelles des mystères égyptiens d'époque gréco-romaine est Plutarque, une opération que Gaëlle Tallet a qualifié de «mise en mythos » ${ }^{15}$. Dans son traité Isis et Osiris, Plutarque affirme que la déesse

incorpora dans les rites les plus sacrés des images, des symboles

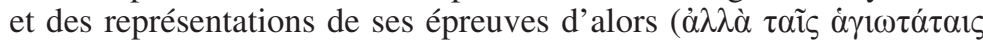

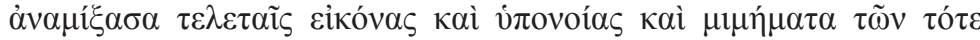
$\pi \alpha \theta \eta \mu \alpha ́ \tau \omega v)$, consacrant en eux une leçon en même temps qu'un exemple de courage pour les hommes et les femmes qu'accableraient

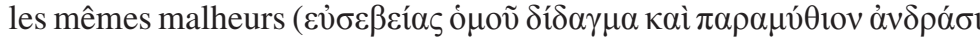

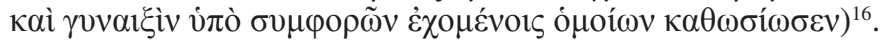

15. Gaëlle Tallet, «Mythologie et hellénisme en Égypte gréco-romaine», Mythos/Fictio. Mythe, imaginaire, fiction, éd. Danièle Auger et Charles Delattre, Nanterre, Presses universitaires de Paris Ouest, 2010, p. 399-425 (http://books. openedition.org/pupo/1847). Sur la conception du mythe égyptien chez Plutarque, voir Youri Volokhine, «À propos de la construction d'un débat sur les mythes égyptiens », Le savoir des religions. Fragments d'historiographie religieuse, éd. Daniel Barbu, Philippe Borgeaud, Mélanie Lozat et al., Gollion, In Folio, 2014, p. 471-498, en part. p. 495-498. Pour une analyse détaillée du traité de Plutarque, avec bibliographie récente, voir Sydney $H$. Aufrère, "Sous le vêtement de lin du prêtre isiaque, le "philosophe" : le "mythe" égyptien comme Sagesse barbare chez Plutarque », Sagesses barbares. Échanges et réappropriation dans l'espace culturel gréco-romain, éd. Sydney H. Aufrère et Frédéric Möri, Genève, La Baconnière, 2016, p. 191-270. Voir en outre l'étude plus ancienne, mais encore utile, de Jean Hani, $L a$ religion égyptienne dans la pensée de Plutarque, Paris, Les Belles Lettres, 1976.

16. Plutarque, Isis et Osiris XXVII, 361E (trad. Christian Froidefond, Collection des Universités de France). 
Les cérémonies rituelles ( $\tau \varepsilon \lambda \varepsilon \tau \alpha i ́)$ conserveraient la trace des «épreuves» $(\pi \alpha \theta \eta \dot{\mu} \mu \alpha \tau \alpha)$ d'Isis. Plutarque ne dit pas que les mystères égyptiens sont construits sur les récits mythiques relatifs à la mort d'Osiris ou à la quête d'Isis, mais il laisse entendre qu'ils ont joué un rôle selon la volonté de la déesse. D'ailleurs, le verbe

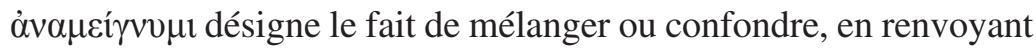
à une mixture d'éléments différents qui constitueraient les rituels célébrés en l'honneur des dieux égyptiens ${ }^{17}$.

Les auteurs chrétiens des $\mathrm{II}^{\mathrm{e}}-\mathrm{IV}^{\mathrm{e}}$ siècles vont au-delà de l'interprétation de Plutarque : ils utilisent le modèle éleusinien, et son mythe fondateur des mystères, et l'appliquent aux mystères égyptiens, pour en faire une catégorie cohérente. Certes, la matrice éleusinienne a été utilisée avant les œuvres des auteurs chrétiens : en effet, l'arétalogie hellénisée de Maronée, en Thrace, présentait déjà à la fin du $\mathrm{II}^{\mathrm{e}}$ siècle avant notre ère une superposition entre Isis et la Déméter d'Éleusis ${ }^{18}$. Mais on était là plus simplement dans le cadre d'une interpretatio entre Isis et Déméter. D'ailleurs, il ne faut pas oublier que lectures grecques et chrétiennes des mystères égyptiens sont étroitement imbriquées: les auteurs chrétiens étaient des intellectuels grecs et romains, qui partageaient la même paideia. Il est donc normal qu'ils réfléchissent aux mystères égyptiens à partir du filtre des mêmes auteurs.

Avant de commencer l'analyse des textes, il est important de rappeler que, dans la construction chrétienne d'une catégorie homogène et unitaire des mystères païens, le mythe trouve une place centrale. Les auteurs chrétiens soulignent, à plusieurs reprises, que «mythes » et «mystères » sont étroitement liés, les seconds ne pouvant pas exister sans les premiers. Lorsque Clément d'Alexandrie s'interroge sur l'origine du mot $\mu v \sigma \tau \hat{\eta} \rho \iota \alpha$, il propose entre autres une explication particulièrement intéressante : "Vous pouvez, autrement, considérer les mystères comme des traditions

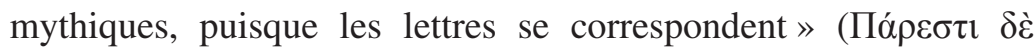

17. Sur cette idée de «mixture », mais dans le cadre de la construction de la statue de Sarapis, voir Nicole Belayche, «Le possible "corps" des dieux : retour sur Sarapis », Dans le laboratoire de l'historien des religions. Mélanges offerts à Philippe Borgeaud, éd. Francesca Prescendi et Youri Volokhine, Genève, Labor et Fides, 2011, p. 227-250, en particulier p. 239-240.

18. Cf. Laurent Bricault, Les cultes isiaques dans le monde gréco-romain, Paris, Les Belles Lettres, 2013, p. 77-80. 


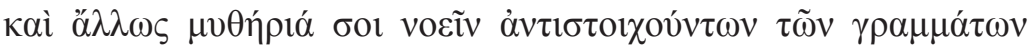

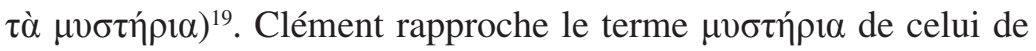
$\mu v \theta \hat{p} \rho \iota \alpha$, qui est un néologisme qu'il faut sans doute considérer comme une invention lexicale de sa part. Le terme sert à produire une assonance avec le diminutif - légèrement péjoratif - $\mu v \theta \alpha ́ \rho ı$, «petits récits», employé notamment pour définir les fables

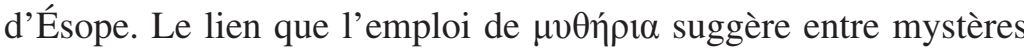
et mythes va permettre à Clément de fonder son interprétation des «mystères païens ». Ce sont en effet des récits mythiques qui sont, pour Clément, à la base de ces pratiques rituelles : c'est pourquoi l'auteur relate tous les mythes fondateurs des $\mu v \sigma \tau \eta ́ p t \alpha$, à savoir la quête et le deuil de Déméter après le rapt de Perséphone, la mise à mort de Dionysos par les Titans, etc.

Plusieurs $\mu v \sigma \tau \eta ́ p ı$ antiques rapportent en effet des mythes étiologiques, des récits qui racontent l'origine de ces pratiques, dont le discours fondateur des mystères d'Éleusis est sans aucun doute le plus célèbre ${ }^{20}$. Lorsqu'ils existent, ces récits restent toutefois à l'arrière-plan dans la célébration des cultes à mystères. Ils sont très probablement connus par les initiés, mais ils n'entrent pas dans la gestualité rituelle, du moins d'après les rares informations dont nous disposons. En revanche, d'après les auteurs chrétiens, les mythes donnent forme à la célébration des mystères, ils en ont façonné le déroulement cultuel.

$\mathrm{Au}$ milieu du $\mathrm{III}^{\mathrm{e}}$ siècle, un autre auteur alexandrin, Origène, paraît confirmer la position de Clément d'Alexandrie, en concentrant plus particulièrement le regard sur les mythes égyptiens. Dans le premier livre de son traité polémique antipaïen, Contre Celse, Origène critique le philosophe Celse, en l'accusant de fonder son interprétation des mythes sur les enseignements des mythes égyptiens :

Que les Égyptiens soient pour Celse les maitres du mythe ( $\mu$ 0 Oov $\delta ı \delta \alpha ́ \sigma \kappa \alpha \lambda o t)$ concernant les embrasements et les inondations, eux qui sont, d'après lui, les plus sages ; les traces de leur sagesse sont des animaux sans raison qui sont vénérés et des discours qui

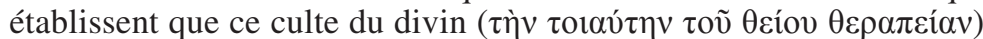

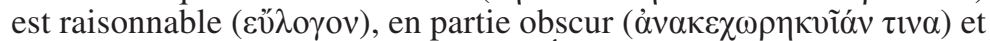
mystérique ( $\mu v \sigma \tau \iota \kappa \eta ́ v)$. [...] Et si les Égyptiens composent des récits mythiques $(\mu v \theta 0 \lambda \mathrm{o} \sigma \tilde{\omega} \sigma \mathrm{t})$, on considère qu'ils ont philosophé par

19. Clément d'Alexandrie, Protreptique II, 13, 2 (traduction personnelle).

20. Il suffit de renvoyer à l'Hymne homérique à Déméter. 


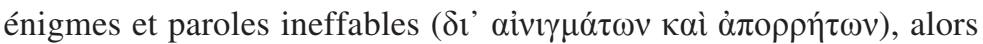

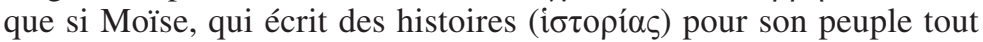
entier, leur laisse des lois, on croit qu'ils sont des récits mythiques vides ( $\mu \tilde{0} \theta$ or $\kappa \varepsilon v o i ́)$, des discours qui n'admettent pas d'allégorie. Ceci est l'opinion de Celse et des Épicuriens ${ }^{21}$.

Les mythes des Égyptiens contiennent une sagesse réservée et non dévoilée qui était propre aux cultes à mystères. Dans la deuxième partie du passage, Origène souligne l'opposition entre

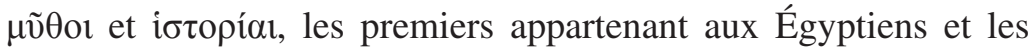
seconds à Moïse. On constate bien le modèle platonicien partagé par les deux auteurs. Mais d'après un auteur chrétien, cette opposition se décline en une opposition entre mythes et Écritures. En même temps, «mythe » et « histoire » se définissent comme des armes de controverses puisque les païens, à leur tour, semblent considérer les i $\sigma \tau$ opíaı de Moïse comme des $\mu \tilde{v} \theta 01$ vides ${ }^{22}$.

\section{MINUCIUS FÉLIX : LE MYTHE FAIT LE MYSTÈre}

Un texte particulièrement instructif sur la question qui nous intéresse est l'Octavius de Minucius Félix. L'ouvrage, qui date de la fin du $\mathrm{II}^{\mathrm{e}}$ siècle ou plus probablement du début du $\mathrm{III}^{\mathrm{e}}$ siècle, est construit comme une compétition entre deux discours sur la vérité, le premier du païen Cécilius et le deuxième du chrétien Octavius. Le dialogue se fonde sur la prétention à la vérité de deux

21. Origène, Contre Celse I, 20 (traduction personnelle).

22. Ibid. $\mathrm{V}, 38$. Au livre $\mathrm{V}$, Origène répond à l'accusation lancée par Celse contre les chrétiens d'avoir abandonné leurs patria, leurs « traditions ancestrales », ces coutumes que chacun doit suivre. Dans ce contexte, il évoque les mythes d'Osiris et Isis : « Énumérer maintenant les récits mythiques sur Osiris et Isis serait superflu et inopportun. Si les récits mythiques étaient exposés allégoriquement, ils nous enseigneraient à vénérer l'eau inanimée et la terre allouée aux hommes et tous les animaux. C'est ainsi, je crois, qu'ils prennent Osiris pour l'eau et Isis pour la terre. À propos de Sarapis, il y a une histoire longue et discordante : il est arrivé hier ou avant-hier grâce aux sorcelleries de Ptolémée qui voulait le représenter aux habitants d'Alexandrie comme un dieu manifeste ( $\dot{\varepsilon} \pi \varphi \omega v \tilde{\eta})$. Nous avons lu chez le pythagoricien Numénius à propos de sa construction ( $\pi \varepsilon \rho i ̀ ~ \tau \tilde{\eta} \varsigma ~ \kappa \alpha \tau \alpha \sigma \kappa \varepsilon v \tilde{\eta} \varsigma)$ qu'il partage l'essence de tous les animaux et les végétaux contrôlés par la nature; il paraît donc avoir été construit comme un dieu par des cérémonies sans effet ( $\mu \varepsilon \tau \grave{\alpha} \tau \tilde{\omega} v \dot{\alpha} \tau \varepsilon \lambda \varepsilon \dot{\sigma} \sigma \omega v \tau \varepsilon \lambda \varepsilon \tau \tilde{\omega} v$ ) et par les sorcelleries qui évoquent des démons, non seulement par des sculpteurs mais aussi par des magiciens, des potions et des démons qui charment par leurs incantations » (traduction personnelle). La lecture allégorique enseigne à voir dans Osiris l'eau et dans Isis la terre. 
personnages et illustre bien la querelle à la fois philosophique et théologique de l'époque ${ }^{23}$.

Dans son réquisitoire contre les cultes païens, Octavius aborde la question des «mystères » des cultes traditionnels, une catégorie qu'il exprime par le couple de termes sacra et mysteria. Plusieurs rituels appartiennent à cette catégorie, d'après le personnage de Minucius Félix : rites liés à Isis et à Osiris, à Cybèle, aux Corybantes, rites éleusiniens. Ce qui réunit toutes ces pratiques, ce sont les récits mythiques qui renvoient à des funérailles, des deuils, des lamentations. L'interprétation n'est pas tout à fait nouvelle sous la plume d'un auteur chrétien: Athénagore d'Athènes avait exprimé l'existence de ce lien vers 170 dans sa Supplique au sujet des chrétiens, en disant que les mystères païens sont les $\pi \alpha \dot{\theta} \eta$, c'est-à-dire les passions et les émotions, des dieux païens ${ }^{24}$. Minucius Félix reprend cette explication, qui deviendra un motif récurrent chez les auteurs chrétiens, notamment de langue latine :

Enfin considère les cérémonies et les mystères eux-mêmes (Considera denique sacra ipsa et ipsa mysteria): tu trouveras des morts sinistres, et des destins funestes, des cérémonies funèbres, des deuils et des lamentations des divinités misérables (inuenies exitus tristes, fata et funera et luctus atque planctus miserorum deorum).

D'après le texte de l'Octavius, c'est le récit mythique qui fait les mysteria, et c'est précisément la condition misérable des divinités païennes qui fonde la liturgie des mystères. On voit bien la volonté d'expliquer les mysteria sur la base du mécanisme mis au point par Évhémère qui voyait dans les dieux la divinisation d'anciens souverains, une stratégie souvent utilisée par les auteurs chrétiens

23. Les deux personnages s'accusent l'un l'autre de ne croire qu'à des mensonges. "Quelles choses incroyables, quels fait prodigieux les chrétiens fabriquent» (Christiani quanta monstra, quae portenta confingunt): c'est Cécilius qui parle, en ajoutant qu'il ne s'agit que de "créations d'une croyance déraisonnable » (figmenta male sanae opinionis) : voir Minucius Félix, Octavius $\mathrm{X}, 5$ et XI, 9. De manière analogue, dans sa réponse, Octavius définit par les termes de figmenta et mendacia les récits que la tradition littéraire ancienne enseignait aux païens depuis l'enfance : Octavius XXIII, 8.

24. Voir Athénagore, Supplique au sujet des chrétiens XXXII, 1. Sur le rapport entre mystères et émotions, voir Philippe Borgeaud, «Rites et émotions. Considérations sur les mystères", Rites et croyances dans les religions du monde romain, éd. John Scheid, Vandœuvres, Fondation Hardt («Entretiens sur l'Antiquité classique », t. LIII), 2007, p. 189-222. 
pour expliquer l'origine des rites traditionnels de l'Empire ${ }^{25}$. Après cette considération générale, Minucius Félix introduit un passage spécifique concernant les mystères d'Isis :

Puisqu'elle a perdu son fils, Isis est en deuil, se lamente, se met à sa recherche avec son cynocéphale et ses prêtres chauves, et les misérables Isiaques se frappent la poitrine et imitent la douleur d'une mère très malheureuse (Isis perditum filium cum Cynocephalo suo et caluis sacerdotibus luget, plangit, inquirit, et Isiaci miseri caedunt pectora et dolorem infelicissimae matris imitantur); bientôt après avoir retrouvé le petit, Isis se réjouit, les prêtres sont enthousiastes, le cynocéphale, qui l'a découvert, se glorifie, et tous les ans ils n'arrêtent pas de perdre ce qu'ils trouvent ou de trouver ce qu'ils perdent (mox inuento paruulo gaudet Isis, exultant sacerdotes, Cynocephalus inuentor gloriatur, nec desinunt annis omnibus uel perdere quod inueniunt uel inuenire quod perdunt). N'est-il pas ridicule d'être en deuil pour ce que tu vénères ou de vénérer ce qui te conduit au deuil ? Néanmoins, ces cérémonies jadis égyptiennes sont maintenant des cérémonies romaines (Nonne ridiculum est uel lugere quod colas uel colere quod lugeas? Haec tamen Aegyptia quondam nunc et sacra Romana sunt $)^{26}$.

À propos de ce passage de l'Octavius, Laurent Bricault a remarqué que Minucius Félix confond «Osiris et Harpocrate, faisant du premier le fils d'Isis alors qu'il n'est que son frère et époux $»^{27}$. Si Minucius Félix identifie Osiris au fils d'Isis, c'est parce qu'il essaie d'uniformiser les mythes égyptiens sur le modèle éleusinien et la quête de Déméter pour retrouver sa fille Coré. D'ailleurs, dans son exposé sur les sacra et les mysteria, Minucius Félix cite, tout de suite après Isis, le mythe de Déméter/ Cérès : "Cérès, entourée de torches allumées et d'un serpent, angoissée, tourmentée, erre çà et là, en quête de Libera qui a été enlevée et souillée : voilà les mystères d'Éleusis » (Ceres facibus accensis et serpente circumdata errore subreptam et corruptam

25. Sur l'évhémérisme, voir Nickolas Roubekas, An Ancient Theory of Religion. Euhemerism from Antiquity to the Present, New York-Londres, Routledge, 2017 et Philippe Borgeaud, "Variations évhéméristes », Les discours sur les religions dans l'Empire romain : regards croisés entre "païens », "juifs » et "chrétiens", éd. Francesco Massa, Revue de l'histoire des religions, t. 234, fasc. 4, 2017, p. 593-612.

26. Minucius Félix, Octavius XXII, 1-2 (traduction personnelle).

27. Laurent Bricault, «Gens Isiaca et identité polythéiste à Rome à la fin du $\mathrm{IV}_{\mathrm{e}}^{\mathrm{s}}$ s. apr. J.-C. », Isis on the Nile. Egyptian Gods in Hellenistic and Roman Egypt, Proceedings of the IV Ith International Conference of Isis Studies (Liège, November 27-29, 2008), éd. Laurent Bricault et Miguel John Versluys, Leyde-Boston, Brill («Religions in the Graeco-Roman World», t. 171), 2010, p. 326-359, ici p. 339. 
Liberam anxia sollicita uestigat : haec sunt Eleusinia $)^{28}$. Le but de Minucius Félix est de faire rentrer les mystères égyptiens dans la catégorie générale des mystères et, pour ce faire, il s'appuie sur le modèle éleusinien qui le conduit à modifier les récits mythiques.

Les mystères égyptiens ne sont donc que l'écho du récit mythique. Dans le mythe, Isis s'afflige, dans les rites mystériques, les prêtres se frappent la poitrine ; Isis se réjouit, les prêtres exultent. D'après Minucius Félix, l'imitation est la clé d'interprétation des rituels célébrés lors des mystères égyptiens ${ }^{29}$.

\section{LACTANCE : « TOUJOURS ILS LE PERDENT, ET TOUJOURS ILS LE RETROUVENT »}

L'idée qu'Osiris est le fils d'Isis revient chez un autre auteur chrétien de langue latine qui appartient à la génération suivante : Lactance. Dans la première partie de ses Institutions divines, achevées au début des années 310 (c'est-à-dire avant la fin de la «Grande persécution» de Dioclétien) ${ }^{30}$, Lactance analyse les religions et les philosophies païennes afin de construire un nouveau savoir chrétien ${ }^{31}$. Il retravaille toutefois son texte à plusieurs reprises et nous savons qu'il a probablement publié deux autres éditions pendant le règne de l'empereur Constantin ${ }^{32}$. Le but de Lactance est de créer une culture et un savoir chrétien global ; pour ce faire, le texte doit nécessairement passer par la condamnation

28. Minucius Félix, Octavius XXII, 2 (trad. Jean Beaujeu, Collection des Universités de France).

29. Sur l'imitation entre rite et mythe, voir Diodore de Sicile IV, 3, 2-3 où l'historien dit que les femmes qui honorent Dionysos «imitent» les ménades du mythe (l'auteur utilise le verbe $\mu \mu \varepsilon \varepsilon_{0} \mu \alpha$ ).

30. Sur la question des persécutions, voir Marie-Françoise Baslez, Les persécutions dans l'Antiquité : victimes, héros, martyrs, Paris, Fayard, 2007.

31. Pour une lecture historique des Institutions divines voir Blandine Colot, Lactance. Penser la conversion de Rome au temps de Constantin, Florence, Léo S. Olschki, 2016. Sur la notion de "paganisme» chez Lactance, voir Francesco Massa, "Lactance, le "paganisme", les dieux : construire l'image de la religion des autres au début du IV ${ }^{\mathrm{e}}$ siècle », La vertu des Païens, Actes du Colloque international transdisciplinaire (Paris, les 17-18 juin 2013), éd. Sylvie Taussig, Paris, Éditions du CNRS, à paraître.

32. Sur les rapports entre Lactance et les empereurs Dioclétien et Constantin, voir Elizabeth DePalma Digeser, The Making of a Christian Empire: Lactantius and Rome, Ithaca, Cornell University Press, 2000. 
des savoirs païens, et notamment des religions et des philosophies traditionnelles.

$\mathrm{Au}$ premier livre, l'auteur aborde la question des sacra et des mysteria présents dans les polythéismes antiques ${ }^{33}$. La formule utilisée ( maintenant il faut dire quelque chose de leurs cérémonies et de leurs mystères », nunc de sacris ac mysteriis eorum pauca dicenda sunt), rappelle ce que Minucius Félix disait dans son Octavius (sacra et mysteria). Lactance propose une sorte de classification de ces cultes, en identifiant trois types différents :

- les sacrifices humains (Jupiter à Chypre; Diane chez les Taures ; les Gaulois ; Jupiter Latiaris ; Saturne ; Jupiter/Saturne à Carthage) ;

- des rites (sacra) violents et sanglants mais qui ne provoquent pas la mort (les publica sacra Matris; les autres sacra en l'honneur de Virtus) ;

- d'autres sacra sans crimes (Isis : Isidis Aegyptiae sacra; Éleusis : sacra Cereris Eleusinae; Priape à Lampsaque ; Hercule : Herculis sacra ; Jupiter en Crète : Cretici Iouis sacra $)^{34}$.

Cette liste met en avant l'une des caractéristiques des Institutions divines puisque l'enjeu, qui dépasse la question des sacra et mysteria, est de créer une taxinomie, de classer des expériences religieuses différentes, dans le cadre d'un examen général du «paganisme $»^{35}$. On voit bien que, dans ce texte, ce que nous appelons «cultes à mystères » est mélangé à d'autres formes cultuelles.

Si l'on regarde plus particulièrement la description de la déesse égyptienne Isis et de ses cultes à mystères, nous pouvons lire :

33. Lactance, Institutions divines I, 21, 1.

34. Ibid. I, 21, 1-49.

35. Sur la notion de «paganisme » comme produit de la réflexion des auteurs chrétiens, voir Maijastina Kahlos, Debate and Dialogue. Christian and Pagan Culture, Aldershot, Ashgate, 2007 ; Alan Cameron, The Last Pagans of Rome, Oxford, Oxford University Press, 2011; Thomas Jürgasch, "Christian and the Invention of Paganism in the Late Roman Empire », Pagans and Christians in Late Antique Rome: Conflict, Competition, and Coexistence in the Fourth Century, éd. Michele Renee Salzman, Marianne Sághy et Rita Lizzi Testa, New York, Cambridge University Press, 2015, p. 115-138. Sur les taxinomies dans le domaine de l'histoire des religions, voir Bruce Lincoln, Discourse and the Construction of Society. Comparative Studies of Myth, Ritual, and Classification, New YorkOxford, Oxford University Press, 1989 et Jonathan Z. Smith, « A Matter of Class: Taxonomies of Religions », Harvard Theological Review, t. 89, fasc. 4, 1996, p. 387-403. 
Il y a les cérémonies égyptiennes pour Isis, puisqu'elle a perdu son jeune fils ou qu'elle l'a retrouvé (Isidis Aegyptia sacra sunt, quatenus filium paruulum uel perdiderit, uel inuenerit). Tout d'abord, en effet, ses prêtres aux corps épilés se battent la poitrine (Nam primo sacerdotes eius, deglabrato corpore, pectora sua tundunt); ils se lamentent, de même qu'elle l'avait fait lorsqu'elle l'avait perdu (lamentantur, sicut ipsa, cum perdidit, fecerat). Ensuite l'enfant est exposé, comme s'il avait été retrouvé, et ce deuil est transformé en joie (Deinde puer producitur quasi inuentus, et in laetitiam luctus ille mutatur) ; pour cette raison, Lucain écrit : «Osiris n'est jamais cherché suffisamment ». En effet, toujours ils le perdent, et toujours ils le retrouvent (Semper enim perdunt, et semper inueniunt $)^{36}$.

Nous remarquons une fois de plus que la construction du mythe d'Isis et Osiris est fondée sur le modèle éleusinien : la perte de l'enfant est l'origine de l'aventure divine, et les retrouvailles donnent lieu à une fête et donc à la célébration du rituel. Chez Lactance, Isis est une mère qui a perdu son fils comme Déméter. L'installation du rituel et son déroulement régulier sont très bien exprimés par la phrase semper enim perdunt, et semper inueniunt, ce qui montre comment le rituel - aux yeux de Lactance - s'était construit sur la succession narrative présente dans les récits mythiques. Dans une démarche qu'on pourrait dire d'historien de religions ${ }^{37}$, Lactance souligne que la pratique rituelle des mystères égyptiens est une « image » de ce qui s'est produit réellement dans le passé ${ }^{38}$.

L'imitation du mythe est à la base de ces mysteria. On pourrait affirmer que les auteurs chrétiens proposent une réflexion sur le lien entre «mythe » et « rite ». D'autre part, le parallèle entre les

36. Lactance, Institutions divines I, 21, 20 (traduction personnelle). L'auteur de la citation n'est pas celui indiqué par Lactance : il ne s'agit pas d'un vers de Lucain, mais d'Ovide (Métamorphoses IX, 693) : voir le commentaire de Pierre Monat, ad loc., Paris, Le Cerf (« Sources Chrétiennes », t. 326), 1986, p. 216.

37. Cf. Lactance, Épitomé XVIII, 5 : «Les cérémonies d'Isis ne montrent rien d'autre que la manière dont elle a perdu et retrouvé son petit garçon appelé Osiris » (Isidis sacra nihil aliud ostendunt nisi quemadmodu filium paruum qui dicitur Osiris perdiderit et inuenerit); trad. Michel Perrin, «Sources Chrétiennes », t. 335. Sur Lactance historien des religions, voir Jean-Claude Fredouille, «Lactance historien des religions ", Lactance et son temps. Recherches actuelles, éd. Jacques Fontaine et Michel Perrin, Paris, Beauchesne, 1978, p. 237-252.

38. Lactance, Institutions divines I, 21, 21 : «On reproduit donc, dans les cérémonies sacrées, une image de ce qui s'est réellement passé » (Refertur ergo in sacris imago rei, quae uere gesta est) ; trad. Pierre Monat, "Sources Chrétiennes », t. 326 . 
récits mythiques de la quête d'Isis et de la quête de Déméter est explicité dans le paragraphe suivant: "De fait les cérémonies de Cérès éleusinienne ne sont pas différentes de ceux-là » (Sacra uero Cereris Eleusinae non sunt his dissimilia) ${ }^{39}$. Même du point de vue de l'articulation des récits mythiques, le modèle éleusinien, avec son mythe, devient la base de la réflexion sur les mystères égyptiens, du moins d'après le regard que les auteurs chrétiens portent sur les mystères égyptiens.

Ce constat permet à Lactance d'introduire l'interprétation qui l'intéresse particulièrement, à savoir l'idée que, si l'événement s'est passé autrefois, cela signifie que «la femme était une mortelle, et presque sans famille, si elle n'avait retrouvé son fils unique » (mortalem mulierem fuisse ac paene orbam, nisi unicum repperisset $)^{40}$. On retrouve donc les motifs de l'évhémérisme, typiques chez les auteurs chrétiens: Osiris est objet d'une consecratio, d'une divinisation après la mort. C'est pourquoi Lactance n'hésite pas à rapprocher le récit concernant Osiris de celui relatif à Romulus ${ }^{41}$.

Un autre élément intéressant du témoignage de Lactance est que l'auteur associe également l'exotisme à l'image des prêtres isiaques : ils ont le corps épilé (deglabrato corpore), ce qui était étranger aux rituels traditionnels romains; c'était par conséquent devenu un cliché de la représentation des prêtres égyptiens. Cette tendance à souligner l'exotisme des cultes orientaux deviendra de plus en plus courante au cours du IV ${ }^{\mathrm{e}}$ siècle, dans le cadre d'un processus de diabolisation du «paganisme» et des cultes à mystères en particulier ${ }^{42}$.

39. Lactance, Institutions divines I, 21, 24 (traduction personnelle).

40. Ibid. (trad. Pierre Monat, « Sources Chrétiennes », t. 326).

41. Lactance, Institutions divines I, 21, 21-23. Cf. Fr. Dunand, « Les mystères égyptiens aux époques hellénistique et romaine », art. cit., p. 48.

42. Sur l'image d'exotisme des cultes orientaux, voir Nicole Belayche, L'altare bilingue. Immigrati orientali e religioni nella Roma imperiale, Bologne, EDB, 2014 ; ead., « Lo sguardo di Roma sull'Oriente », Roma città degli dèi. La capitale dell'impero come laboratorio religioso, éd. Corinne Bonnet et Ennio Sanzi, Rome, Carocci, 2018, p. 327-342. Sur l'interprétation chrétienne de ce même exotisme, voir dans le même volume Francesco Massa, «Lo sguardo cristiano sui culti orientali », p. 343-354 et L. Bricault, « Gens isiaca et identité polythéiste à Rome à la fin du IV s. apr. J.-C. », art. cit. 


\section{Firmicus MATERnUS : UNE HiSTOIRE DE FAMILLE SORDIDE}

Le dernier auteur que je souhaite prendre en considération ici est Firmicus Maternus. D'abord auteur d'un traité d'astrologie, la Mathesis, Firmicus Maternus rédige vers les années 340 une œuvre polémique contre les cultes traditionnels de l'Empire romain, L'erreur des religions païennes, sous le règne des trois fils de Constantin (Constant I ${ }^{\text {er }}$, Constantin II et Constance II). Cette période représente une étape ultérieure dans le renversement des positions d'autorité entre païens et chrétiens. Les nouveaux empereurs poursuivent et intensifient l'activité de Constantin dans la concession de bénéfices aux églises; et surtout, ils inaugurent une politique explicite de limitation de la liberté de culte pour les païens ${ }^{43}$. Certes, les dispositions, par la suite rassemblées dans le livre XVI du Code Théodosien, n'impliquent pas directement l'interdiction de toute forme de ritualité traditionnelle, mais elles témoignent du processus qui a conduit à la promulgation de l'édit de Thessalonique en 380, édit qui fit du christianisme des évêques de Rome et d'Alexandrie la religion officielle de l'Empire et à l'interdiction générale du paganisme en 390-39144.

L'une des originalités de L'erreur des religions païennes de Firmicus Maternus est d'orienter la polémique antipaïenne principalement contre les cultes qui sont considérés, chez les Romains, comme d'origine orientale. Si l'on regarde la deuxième partie du traité qui se focalise presque uniquement sur les formules sacrées (les symbola) des cultes à mystères, aucune mention n'y est faite des cultes romains. Toute la place est occupée par

43. Cf. Code Théodosien XVI 10, 2 (Constance II condamne la superstitio et l'insania sacrificiorum) et XVI 10, 4 (le même empereur ordonne la fermeture des temples et l'abolition des sacrifices). À ce propos, voir Jean Gaudemet, Paolo Siniscalco et Gian Luigi Falchi, Legislazione imperiale e religione nel IV secolo, Rome, Istituto Patristico Augustinianum, 2000. Plus généralement, voir Alessandro Saggioro, La religione e lo stato: cristianesimo e alterità religiose nelle leggi di Roma imperiale, Rome, Bulzoni, 2011.

44. Cf. Code Théodosien XVI 1, 2 (édit de Thessalonique) et XVI, 10, 10-12 (lois antipaïennes de 391-392). Sur ces textes de lois et leur commentaire, voir Les lois religieuses des empereurs romains de Constantin à Théodose II (312-438), vol. I. Code théodosien livre XVI, texte latin de Theodor Mommsen, traduction de Jean Rougé, introduction et notes de Roland Delmaire, avec la collaboration de François Richard, Paris, Le Cerf (« Sources Chrétiennes », t. 497), 2005, p. 439-442. 
les «cultes orientaux» (Mithra, Cybèle et Attis, Isis et Osiris), auxquels s'ajoute la figure du dieu grec Dionysos dont on connaît la prétendue origine étrangère dès l'époque classique grecque ${ }^{45}$.

Les mystères égyptiens sont abordés à plusieurs reprises dans le texte de Firmicus, mais la section la plus intéressante pour notre propos se trouve au chapitre II. Firmicus reconnaît l'existence de quatre éléments fondamentaux dans le monde: le feu, l'eau, l'air et la terre. Et il s'oppose aux peuples (gentes) qui donnent la primauté à l'un de ces éléments et qui en font un « dieu suprême » (summus deus). C'est le cas, par exemple, des Égyptiens et de leur vénération de l'eau. Déjà Diodore de Sicile avait attribué aux Égyptiens la définition d'une sorte de «théologie des éléments » ${ }^{46}$. Selon moi, pour comprendre ce passage de Firmicus, il est nécessaire d'interpréter cette polémique portant sur l'eau rituelle à la lumière des compétitions entre païens et chrétiens autour du rite du baptême. À une époque où les diverses Églises chrétiennes essaient de définir une liturgie baptismale propre, la confrontation (et l'opposition) avec les rituels païens et juifs qui utilisaient l'eau était fondamentale. L'identité rituelle du baptême ne pouvait pas se préciser sans une différenciation avec les autres pratiques analogues qui existaient dans les territoires de l'Empire ${ }^{47}$.

D'après Firmicus, les mystères égyptiens (on retrouve les termes latins sacra et mysteria, exactement comme chez Minucius Félix

45. Sur le lien entre Dionysos et l'Orient dans le débat historiographique et chez Firmicus Maternus, voir Francesco Massa, « Pourquoi Bacchus? L'appendice sur les mystères bachiques à Rome dans la construction herméneutique des Religions orientales de Franz Cumont », The Christian Mystery. Early Christianity and the Pagan Mystery Cults in the Work of Franz Cumont (1868-1947) and in the History of Scholarship, éd. Annelise Lannoy et Danny Praet, Stuttgart, Franz Steiner, à paraître.

46. Diodore de Sicile I, 12, 1 : «Or les Égyptiens prirent chacun de ces éléments pour des dieux et, pour chacun d'entre eux, les premiers hommes qui, en Égypte, firent usage d'un langage articulé trouvèrent un nom particulier en rapport avec son

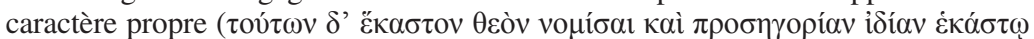

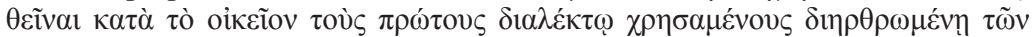

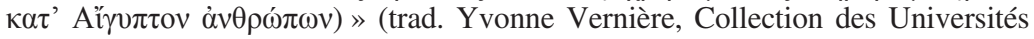
de France).

47. Il suffit de renvoyer au traité d'Ambroise, Des sacrements II, 2 : «Il y a beaucoup d'espèce de baptêmes, mais il n'y a qu'un seul baptême, s'écrie l'Apôtre $[$ Eph 4,5$]$. Pourquoi ? Il y a les baptêmes des païens, mais ce ne sont pas des baptêmes. Ce sont des ablutions, ce ne peuvent être des baptêmes » (Multa genera baptismatum, sed unum baptisma clamat apostolus. Quare? Sunt baptismata gentium, sed non sunt baptismata. Lauacra sunt, baptismata esse non possunt) ; trad. Bernard Botte, « Sources Chrétiennes », t. 25. 
et Lactance $)^{48}$ se fondent encore une fois sur les mythes d'Isis et Osiris :

Mais dans leurs cérémonies qu'ils appellent «mystères », ils ajoutent des funérailles tragiques et <des conflits épouvantables> porteurs d'un malheur funeste (Sed in sacris suis quae mysteria uocant addunt tragica funera et funestae calamitatis <metuenda certamina $>$ ) : l'inceste et l'adultère commis avec sa sœur, et l'action criminelle vengée par les punitions sévères du mari. Isis est la sœur, Osiris le frère, Typhon le mari (incestum cum sorore adulteriumque commissum, et hoc facinus seueris mariti animaduersionibus uindicatum. Isis soror est, Osyris frater, Tyfon maritus $)^{49}$.

Comme souvent chez Firmicus, non seulement les récits mythiques antiques sont soumis au filtre de l'évhémérisme, mais sont aussi banalisés et tournés en ridicule. Dans cet extrait, le mythe des mystères égyptiens devient une sorte d'histoire de famille sordide. Ce qui compte pour l'auteur chrétien est de discréditer les faux mysteria, puisqu'on assiste à une situation de conflit et de concurrence entre les vrais mystères chrétiens et ces cérémonies qu'ils appellent des mystères.

C'est le verbe addere, "ajouter», qui me parait essentiel pour notre propos. Dans la perspective de Firmicus Maternus, les mysteria sont des rites dans lesquels les païens ont ajouté des récits mythiques. Dans L'erreur des religions païennes, le regard chrétien est moins centré sur la question de l'imitation que sur l'idée des mystères païens comme une composition bâclée. Robert Turcan a souligné que «Firmicus Maternus revient plusieurs fois sur cette idée que le paganisme est fait d'incrustations adventices, d'additions qui couvrent une marchandise suspecte $»^{50}$.

Isis et Osiris ne sont plus mère et fils, comme chez Minucius Félix et Lactance. Dans la version du mythe proposée par Firmicus Maternus, Isis est l'épouse de Typhon, mais elle cède à la passion incestueuse de son frère Osiris :

48. À la différence de Minucius Félix et Lactance, Firmicus Maternus n'utilise le terme mysteria que deux fois dans son traité antipaïen : la première occurrence est celle citée ici pour définir les rituels égyptiens ; la seconde, au singulier, est utilisée pour parler du mystère chrétien (L'erreur des religions païennes XIX, 5-6).

49. Firmicus Maternus, L'erreur des religions païennes II, 1 (traduction personnelle). Sur ce passage, voir l'édition critique de Robert Turcan, Firmicus Maternus, L'erreur des religions païennes, Paris, Les Belles Lettres, 2002, p. 170-174 et Paolo Scarpi, Le religioni dei misteri, t. II, Milan, Mondadori, 2002, p. 503-504.

50. R. Turcan, op. cit., p. 173. 
Lorsqu'il apprit qu'Isis, sa femme, avait été corrompue par les désirs incestueux de son frère, il [scil. Typhon] tua Osiris et le déchira par morceaux, et il abandonna les membres palpitants de son misérable corps sur toutes les rives du fleuve Nil (Is cum comperisset Isidem uxorem incestis fratris cupiditatibus esse corruptam, occidit Osyrim artuatimque lacerauit, et per omnes Nili fluminis ripas miseri corporis palpitantia membra proiecit). Après avoir répudié Typhon pour ensevelir son frère et amant, Isis s'adjoignit Nephthys, sa sœur, et Anubis, un chasseur à qui est attribuée une tête de chien puisqu'il découvrit les parties du corps déchiré, à l'aide d'un chien qui suit les traces (Isis repudiato Typhone, ut et fratrem sepeliret et coniugem, adhibuit sibi Nepthum sororem sociam et Anubem uenatorem, cui deo caninum caput impositum est quia lacerati corporis partes artificio canis uestigentis inuenit). Ainsi, Osiris retrouvé, Isis lui donna une sépulture (Sic inuentum Osyrim Isis tradidit sepulturae ${ }^{51}$.

Le récit mythique se transforme en une sorte de feuilleton pathétique. L'union entre Isis et Typhon n'est pas attestée dans les sources dont nous disposons, ni dans la documentation égyptienne ; c'est là un pur travestissement du mythe dû à Firmicus. Plutarque parle d'une union entre Osiris et la femme de Typhon, Nephthys (et les textes égyptiens avant lui), mais il n'est pas question de ce récit dans le traité chrétien ${ }^{52}$. Firmicus modifie le mythe afin de présenter Isis non seulement comme soumise au crime de l'inceste, mais aussi comme une épouse adultère. Son but est de mettre au centre de cette nouvelle version du mythe l'immoralité d'Isis. On pourrait presque affirmer que Firmicus christianise le mythe d'Isis et Osiris pour qu'il soit plus scandaleux au filtre de la morale chrétienne ${ }^{53}$.

Dans son interprétation du mythe fondateur des mystères égyptiens, Firmicus refuse toute lecture allégorique du mythe d'Isis et Osiris :

Mais voici que sur ces funérailles et ces deuils (Sed in his funeribus et luctibus) [il s'agit réellement de funérailles qui ont été célébrées et dont les vestiges subsistent actuellement, car il existe en Égypte un tombeau d'Osiris, et l'on y voit les restes de son cadavre brûlé] (quae uere sunt funera quae facta sunt, quorum extant hodieque reliquiae - nam et sepulchrum Osyridis hodieque in Aegypto est, et cremati

51. Firmicus Maternus, L'erreur des religions païennes II, 1 (traduction personnelle).

52. Plutarque, Isis et Osiris XIV, 356F. Sur cela voir R. Turcan, op. cit., p. 174.

53. Cf. P. Scarpi, Le religioni dei misteri, t. II, p. 480 : « Il passo testimonia l'esistenza di una interpretazione cristiana del mito di fondazione ». 
corporis reliquiae cernuntur-) les défenseurs de ces rites veulent ajouter une explication "physique », en alléguant qu'Osiris représente les semences des moissons, Isis la terre, Typhon la chaleur (defensores eorum uolunt addere physicam rationem, frugum semina Osyrim dicentes esse, Isis terram, Typhonem calorem $)^{54}$.

On voit bien que le problème tourne ici autour des reliquiae d'Osiris, des vestiges du corps d'Osiris qui sont conservés dans un (ou plusieurs) sanctuaire(s) en Égypte et qui sont l'objet d'un culte $^{55}$. Mais Firmicus s'oppose plus particulièrement à la physica ratio qui, mise au point notamment par les stoïciens et reprise par les néoplatoniciens de l'époque impériale, n'est pas acceptable aux yeux de Firmicus ${ }^{56}$. Ici émerge la dimension philosophique du discours de Firmicus. Cette accusation contre la physica ratio n'est pas anodine. Quelques années plus tard, sans doute vers 362, Sallustius, philosophe proche de l'empereur Julien, consacre une réflexion importante sur les mythes, en proposant une taxinomie intéressante: d'après Sallustius les mythes seraient divisés en cinq catégories - théologiques, physiques, psychiques, matériels, mixtes ${ }^{57}$. Les mythes égyptiens correspondraient aux mythes matériels, à savoir les moins nobles. Selon lui,

Matériel, et de dernière valeur, est le genre de mythes qu'en raison de leur absence d'instruction employèrent surtout les Égyptiens, eux qui ont tenu les corps eux-mêmes pour des dieux et ont appelé la terre Isis, l'humidité Osiris, la chaleur Typhon, ou l'eau Cronos,

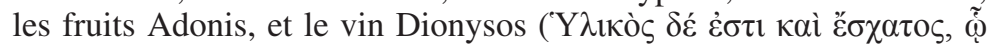

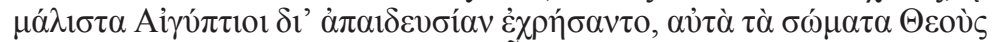

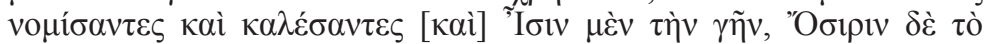

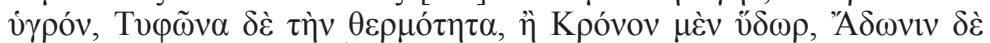

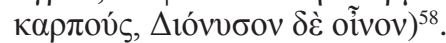

Il est clair qu'à cette époque l'interprétation allégorique des mythes était associée aux Égyptiens, et elle était aussi une raison

54. Firmicus Maternus, L'erreur des religions païennes II, 6 (traduction R. Turcan).

55. Sur les « reliques » d'Osiris dans la religion égyptienne, voir Laurent Coulon, «Les reliques d'Osiris en Égypte ancienne : données générales et particularismes thébains ", Les objets de la mémoire. Pour une approche comparatiste des reliques et de leur culte, éd. Philippe Borgeaud et Youri Volokhine, Berne-Berlin-Bruxelles, Peter Lang, 2005, p. 15-46.

56. R. Turcan, op. cit., p. 183-184.

57. Sallustius, Des dieux et du monde IV, 1.

58. Ibid. IV, 3 (trad. Gabriel Rochefort, Collection des Universités de France). 
du conflit entre païens et chrétiens. L'opération de Firmicus a donc un double enjeu : d'une part, elle vise à opposer vrais et faux mystères, à savoir mystères chrétiens et païens, mais d'autre part, elle essaie de combattre les lectures allégoriques et philosophiques qui étaient faites des cultes à mystères depuis l'époque classique.

\section{Conclusions}

On remarquera tout d'abord que les sources chrétiennes qui développent la question des «mystères égyptiens » sont notamment des sources en latin, alors que généralement, ce sont les chrétiens de langue grecque qui s'intéressent aux cultes à mystères grecs et romains, entre autres parce que le vocabulaire mystérique est essentiellement un vocabulaire grec (mystèria, teletai, orgia, etc.) que les auteurs latins se contentent généralement de traduire par le terme sacra, « les cérémonies ».

Étant donné le nombre limité de témoignages sur les mystères égyptiens, il est évident que les sources chrétiennes représentent des documents précieux pour analyser et interpréter ces cultes. Toutefois, le parcours que j'ai essayé de tracer invite à la prudence. Pour les auteurs chrétiens, les mystères illustrent les mythes d'Isis (ou d'autres divinités) ou ce qu'ils veulent en savoir, puisqu'ils les modifient à leur guise au mépris de la logique des Autres. Il convient aussi de relire et situer cette association entre «mythe » et «mystères » faite par les auteurs chrétiens dans leur dimension polémique et apologétique. Il convient de rappeler qu'à cette époque une réelle compétition rituelle existait à propos de ce que les chrétiens et les païens appelaient les mystères. Si les mystères païens sont simplement le résultat de récits mythiques honteux et scandaleux, ils deviennent soudainement moins dangereux dans la compétition avec les mystères chrétiens.

Dans cette perspective, je pense qu'il faut éviter de faire des « mystères égyptiens » des rituels qui mettent simplement en scène les mythes d'Isis et Osiris. L'absence de cette dimension dans les sources païennes, comme Plutarque et Apulée, n'est pas sans importance. C'est sans doute une banalisation et une déformation voulue par les auteurs chrétiens. En revanche, il est important de 
souligner que l'association de «mythe » et «mystères » entre davantage dans la tendance chrétienne des premiers siècles à construire une catégorie unique de «mystères païens» plus facilement opposable aux vrais mystères chrétiens.

Francesco.Massa@unige.ch 